\title{
85701 - USO DE FÁRMACOS EM IDOSOS INSTITUCIONALIZADOS: POLIFARMÁCIA, MEDICAMENTOS INAPROPRIADOS E DUPLICIDADE TERAPÊUTICA
}

\author{
Apresentação Oral - Geriatria
}

\author{
Marion Dors Perotti / Perotti, MD / ULBRA CANOAS; \\ Paulo Roberto Cardoso Consoni / Consoni, PRC / ULBRA CANOAS
}

Introdução: o envelhecimento populacional é fato no Brasil,aumentando a prevalência de doenças crônicas, em especial as demenciais e neurológicas, que demandam maior cuidado dos idosos em instituições. Sabe-se que a população idosa residente em instituição de cuidado é considerada mais doente do que a da comunidade, o que amplia a probabilidade de erros de prescrição ${ }^{1}$, uso excessivo de fármacos ou sobreposição dos mesmos. Sendo assim, é necessário analisar com que frequência tais fatos ocorrem, para que mudanças sejam implementadas, diminuindo os erros em prescrições de idosos ${ }^{2},{ }^{3}, 4$. Objetivos: avaliar o número de fármacos utilizado por idoso, a prevalência de polifarmácia, medicamentos inapropriados e duplicidade terapêutica. Métodos: estudo transversal, realizado em residencial geriátrico de Canoas, RS. Foram analisados os 42 prontuários do local.Variáveis: número de medicamentos prescritos, classe farmacológica mais utilizada e interações medicamentosas.Sexo, idade e o diagnóstico principal dos pacientes também foram analisados.O estudo teve aprovação no Comitê de Ética e Pesquisa sob o número 3.361.728. Resultados: dentre os 42 pacientes avaliados, 15 eram homens e 27 mulheres. A média de idade foi de 68 anos. O diagnóstico mais frequente foi Sequela de Acidente Vascular Cerebral não especificado, encontrado em 14 pacientes. A média de medicamentos utilizado por pessoa foi de 4.9, sendo que 23 pacientes apresentavam polifarmácia. Os medicamentos mais utilizados foram do aparelho cardiovascular (35\%). Identificaram-se 87 interações potenciais em 12 pacientes e duplicidade terapêutica em 2 pacientes. $\mathrm{O}$ medicamento potencialmente inapropriado mais utilizados foi o alprazolam (4,3\%).Conclusões: tendo em vista que os achados influenciam na qualidade de vida do idoso, é fundamental a avaliação do plano terapêutico.Por fim, prezar por uma farmacoterapia racional e segura é expressão de qualidade na assistência ao idoso.

Palavras-chave: idoso, prescrição de medicamentos, polifarmácia.

Referências: 1. Dantas MS, Santos VC. Implicações da polifarmácia entre idosos. LEFD; 2018; 2. Egger SS, et al. Prevalence of potentially inappropriate medication use in elderly patients 2006; 3. Heider D, Matschinger H, Meid DA. The impact of potentially inappropriate medication on the development of health care costs and its moderation by the number of prescribed substances. PLoS ONE. 2018; 4. Scott IA, Hilmer SN, Reeve E et al. Reducing inappropriate polypharmacy. JAMA In. 2015 